\title{
Holographic superconductors in the AdS black hole with a magnetic charge
}

\author{
M. R. Setare $, 1, *$ D. Momeni, ${ }^{2,+}$ R. Myrzakulov, ${ }^{2,}+$ and Muhammad Raza, $3, \S$ \\ ${ }^{1}$ Department of Science, Payame Noor University, Bijar, Iran \\ ${ }^{2}$ Eurasian International Center for Theoretical Physics, \\ Eurasian National University, Astana 010008, Kazakhstan \\ ${ }^{3}$ Department of Computer Science, COMSATS Institute of \\ Information Technology (CIIT), Sahiwal campus, Pakistan
}

\begin{abstract}
In this work we study the analytical properties of a $2+1$ dimensional magnetically charged holographic superconductor in $A d S_{4}$. We obtain the critical chemical potential $\mu_{c}$ analytically, using the Sturm-Liouville variational approach. Also, the obtained analytic result can be used to back up the numerical computations in the holographic superconductor in the probe limit.

PACS numbers: $11.25 . \mathrm{Tq}$
\end{abstract}

Keywords: High- $T_{C}$ superconductors theory

\section{INTRODUCTION}

In this paper, we will investigate the holographic superconductors in magnetically charged planar AdS black hole. The metric of the black hole with a magnetic charge was obtained by Romans 1]. The presence of the magnetic charge shows that the black hole has different horizon structure from that of the uncharged Schwarzschild black hole. The main purpose in this paper is to see how the magnetic charge affects the holographic superconductors in this asymptotic AdS black hole. This paper is organized as follows: In Sec. II, we present the metric describing a magnetically charged planar Schwarzschild-AdS black hole. In Sec. III, we give the basic equations. In Sec. IV we investigate the zero temperature limit and critical chemical potential. We study analytically holographic superconductors in the magnetically charged planar black hole background. Our results show that the magnetic charge presents the different physical effects on the different condensations. Finally, in the last section we present our conclusions.

\footnotetext{
*Electronic address: rezakord@ipm.ir

${ }^{\dagger}$ Electronic address: d.momeni@yahoo.com

${ }^{\ddagger}$ Electronic address: rmyrzakulov@csufresno.edu

${ }^{\S}$ Electronic address: mreza06@gmail.com
} 


\section{GEOMETRY OF A MAGNETICALLY CHARGED BLACK HOLE IN $A d S_{4}$}

The Lagrangian that gives rise to the magnetically charged black hole is described by the common Lagrangian of Einstein-Maxwell theory with cosmological constant $\Lambda$ is given by [1]

$$
\mathcal{L}=\frac{1}{q}\left(-\frac{1}{4} R+\frac{1}{4} F_{\mu \nu} F^{\mu \nu}+\frac{1}{2} \Lambda\right)
$$

Where $q$ is the electrical charge. The usual Einstein-Maxwell equations read

$$
R_{\mu \nu}=T_{\mu \nu}, \quad T_{\mu \nu}=2 F_{\mu \alpha} F_{\nu}^{\alpha}-\frac{1}{2} g_{\mu \nu} F_{\alpha \beta} F^{\alpha \beta}, \quad \nabla_{; \mu} F^{\mu \nu}=0
$$

The components of the Maxwell tensor are

$$
F_{01}=\frac{Q}{r^{2}}, \quad F_{23}=-H \cos \theta
$$

We assume that the electric charge $Q$ and the magnetic charge $H$ simultaneously are not zero. The magnetically charged planar solution for action (1) is [1]

$$
d s^{2}=-f(r) d t^{2}+\frac{d r^{2}}{f(r)}+r^{2}\left(d x^{2}+d y^{2}\right) .
$$

An exact solution of the equations (2) is

$$
f(r)=r^{2}-\frac{M}{r}+\frac{H^{2}}{r^{2}}
$$

This solution describes a planar Schwarzschild-AdS black hole with a magnetic charge. As the magnetic charge $H$ tends to zero, the space time reduces to a four dimensional SchwarzschildAdS black hole. The only unique non vanishing component $F_{x y}=\frac{H}{r^{2}}$. Thus the factor $H$ can be interpreted as the strength of the magnetic field in bulk. It is obvious that this solution is asymptotically $A d S_{4}$. The outer horizon locates at $f(h)=0$. For future numerical purposes, we take the horizon as $h=1$. Thus we have $M=1+H^{2}$. Further, as we know, to have a real horizon we must satisfy the auxiliary condition

$$
27 M^{4}-256 H^{6} \geq 0
$$

We limit ourselves only to the extremal black hole, i.e., such values of the magnetic charge satisfying

$$
27 M^{4}-256 H^{6}=0
$$

which gives to us the value of

$$
H=1.73205
$$


- This is the extremal magnetic field which leads to an extremal black hole. The black hole Hawking-Unruh temperature reads as

$$
T_{B H}=\frac{f^{\prime}(h)}{4 \pi}
$$

This temperature can be read as the boundary temperature in the quantum dual theory, via CFT. We mention here that the near horizon geometry of the metric, at zero temperature has a scaling invariance. This scaling invariance characterized by a dynamical critical exponent $z$.

\section{THE CONDENSATE OF CHARGED OPERATORS}

In order to investigate the holographic superconductors in the background of the planar Schwarzschild-AdS black hole with a magnetic charge, we need a scalar condensate with a charged scalar field $\psi[2]$. Here we work in the probe approximation by neglecting the backreaction of the charged scalar field $\psi$. It may be an interesting topic to study the holographic superconductors of the scalar field with magnetic charge. However, In this paper we only consider the condensate of an external charged scalar field in the background of a black hole with a magnetic. Let us consider a Maxwell field and a charged complex scalar field,

$$
S=\int \sqrt{-g} d^{4} x\left[\frac{1}{q}\left(-\frac{1}{4} R+\frac{1}{4} F_{\mu \nu} F^{\mu \nu}+\frac{1}{2} \Lambda\right)-\left|D_{\mu} \psi\right|^{2}-m^{2}|\psi|^{2}\right] .
$$

We set the AdS radius $L=\sqrt{\frac{3}{\Lambda}} \equiv 1$. The mass of the scalar field is chosen such that it remains below to the Breitenlohner-Freedman bound [3]. We take

$$
A_{\mu}=(\phi(r), 0,0,0), \quad \psi=\psi(r),
$$

we can obtain the equations of motion for the complex scalar field $\psi$ and electrical scalar potential $\phi(r)$ in the background of the Schwarzschild-AdS black hole with a magnetic charge $H$ given by [4]

$$
\begin{array}{r}
\psi^{\prime \prime}+\left(\frac{f^{\prime}}{f}+\frac{2}{r}\right) \psi^{\prime}+\left(\frac{\phi^{2}}{f^{2}}+\frac{2}{f}\right) \psi=0 \\
, \phi^{\prime \prime}+\frac{2 \phi^{\prime}}{r}=\frac{2 \psi^{2} \phi}{f} .
\end{array}
$$

Here a prime denotes the derivative with respect to $r$. Trivially, it is not possible to obtain the nontrivial analytical solutions to the nonlinear equations. 


\section{ZERO TEMPERATURE LIMIT AND CRITICAL CHEMICAL POTENTIAL}

We analyze the field equations using the variational method. On horizon the boundary condition $\phi(h)=\mu$ is the critical chemical potential reads from the value of the field on boundary (horizon), and for the scalar field $\psi^{\prime}(h)=v(h, H) \psi(h)$ in which the function $v(h, H)$ is a function of $h, H$. Asymptotic value of the field $\psi$ leads to two distinct conformal dimensions $\Delta_{ \pm}=1,2$. The first step for investigating the analytical properties of the superconductors via variational method is in writing the field equation in a classical Sturm-Liouville (S-L) form. We present our calculations separately for different conformal dimensions. We rewrite the field equations (7) and (8) in a new coordinate $z$ as

$$
\begin{aligned}
\psi^{\prime \prime}+\frac{f^{\prime}}{f} \psi^{\prime}+\left(\frac{h^{2} \phi^{2}}{z^{4} f^{2}}+\frac{2 h^{2}}{z^{4} f}\right) \psi & =0 \\
\phi^{\prime \prime}-\frac{2 h^{2} \psi^{2}}{f z^{4}} \phi & =0 .
\end{aligned}
$$

now prime denotes the derivative with respect to the $z$. In these coordinates $z \rightarrow 0$ will be the $A d S_{4}$ conformal boundary. We must first find a solution to the above equations such that near the boundary $\phi \approx \frac{\langle O>}{z^{2}}$ is purely normalizable. Away from the boundary, the solution should end at a regular black hole horizon at $r=h$. Beside the critical point $T=T_{c}, \mu=\mu_{c}$ we have $\phi=\mu(1-z)$, also since in this point

$$
\psi \sim<O>z^{\Delta} F(z)
$$

which in that the new function $F(z)$ satisfies the following Sturm-Liouville equation

$$
\frac{d}{d z}\left(p(z) F^{\prime}\right)+q(z) F=-\mu^{2} w(z) F
$$

here

$$
\begin{array}{r}
f=\frac{h^{4}-h M z^{3}+H^{2} z^{4}}{h^{2} z^{2}} \\
p(z)=z^{2 \Delta} f \\
q(z)=\Delta(\Delta-1) z^{2 \Delta-2} f+\Delta z^{2 \Delta-1} f^{\prime}+2 h^{2} z^{2 \Delta-4} \\
w(z)=\frac{h^{2} z^{2 \Delta-4}(1-z)^{2}}{f} .
\end{array}
$$

It's easy to show that the minimum value of the eigenvalue $\mu^{2}$ which gives the minimum of the magnetic field $\mathrm{H}$ can be obtained from the following functional

$$
\mu^{2}=\operatorname{Min}\left\{-\frac{\int_{0}^{1}\left(p(z) F^{\prime 2}-q(z) F^{2}\right) d z}{\int_{0}^{1} w(z) F^{2} d z}\right\} .
$$


The boundary condition is $\left.p(z) F(z) F^{\prime}(z)\right|_{0} ^{1}=0$. From the limit of this boundary term in $z=0$ we observe

$$
\lim _{z \rightarrow 0} p(z) F(z) F^{\prime}(z)=0
$$

Using eq. (16) to compute the minimum eigenvalue of $\mu^{2}$ for $\Delta_{i}, i=+$ or $i=-$, we can obtain the critical temperature $T_{c}$. We have to consider $F(0)=0$ or $F^{\prime}(0)=0$. The trial function $F(z)=1-\alpha z^{2}$ is a good choice satisfying this restricted boundary condition $F^{\prime}(0)=0, F(0)=1$. We analyze two cases $\Delta_{ \pm}=1,2$ separately.

\section{A. Case $\Delta=2$}

In this case we have

$$
\mu^{2}(\alpha)=-\frac{1.333 \alpha^{2}-0.1904 \alpha+0.4}{0.0289 \alpha^{2}-0.0873 \alpha+0.0858}
$$

The minimum with respect to the $\alpha$ locates at

$$
\alpha_{c}=-1.75697
$$

which leads to the following values for $\mu_{c}$

$$
\mu_{c}=1.86784
$$

we show that the $\mu_{c}$ obtained from variational method is in good agreement with the results of the [4].

\section{B. Case $\Delta=1$}

In this case, we have

$$
\mu^{2}(\alpha)=-\frac{0.190476 \alpha^{2}+0.4 \alpha}{0.101774 \alpha^{2}-0.171785 \alpha+0.43521} .
$$

The minimum with respect to the $\alpha$ locates at

$$
\alpha_{c}=-0.780319,
$$

which leads to the following values for $\mu_{c}$

$$
\mu_{c}=0.55744
$$


Referring to the numerical results obtained in [4] shows that the $\mu_{c}$ obtained is in good agreement with the results. The consistency between the analytic and numerical results indicates that the S-L method is a powerful analytic way to investigate the holographic superconductor even when we take the backreaction into account [5].

\section{CONCLUSION}

In this paper using an variational calculation we obtained the minimum of the chemical potential $\mu_{c}$. Our analytic result can be used to back up the numerical computations in the holographic superconductor in the probe limit.

\section{COMMENT}

The model and the method which we used here are completely different from the published paper [6] in which they applied numerical methods on global monopoles in the AdS background.

\section{ACKNOWLEDGMENT}

We thank Prof. Alberto Salvio for usefull suggestions.

[1] L. J. Romans, Nucl. Phys. B 383, 395 (1992), arXiv:hep-th/9203018.

[2] G. T. Horowitz and M. M. Roberts, Phys. Rev. D 78126008,(2008).

[3] P. Breitenlohner, D. Z. Freedman, Annals Physics 144 249,(1982).

[4] E. Nakano, W.-Y. Wen, Phys. Rev. D 78, 046004 (2008).

[5] Q. Pan, J. Jing, B. Wang, S. Chen, JHEP06,087(2012).

[6] S. Chen, L. Wang, C. Ding, J. Jing, arXiv:0912.2397. 